\title{
Efficient Testing of RF MIMO Transceivers Used in WLAN Applications
}

\author{
Erkan Acar \\ and Sule Ozev \\ Duke University \\ Durham, NC \\ $\{$ ea5, sule $\} @$ ee.duke.edu
}

\begin{abstract}
Multiple input multiple output (MIMO) systems that enable data transfer rates beyond $100 \mathrm{Mbps}$ for WLAN applications is under development and will emerge rapidly. While the higher data rates will enable numerous features in modern communication devices, the industry has to face the soaring test cost due to the increased test complexity and test time. Since MIMO systems have multiple RF paths, each path needs to be individually tested. Moreover, mismatch parameters among the multiple paths require synchronized sampling of RF signals, requiring complicated equipment. In this paper, we propose a low cost MIMO test solution that targets the specifications that are fundamental for MIMO operation, such as gain, IIP3, and phase imbalances between the RF paths. Our test methodology measures these parameters with a high accuracy while requiring only one RF frequency synthesizer. Using the proposed test method, RF MIMO systems can be tested using a mixed signal tester and a single fixed frequency RF signal generator.
\end{abstract}

\section{INTRODUCTION}

Increased performance expectations in the communications market drive the industry towards implementing cost effective solutions to meet consumer demands. Recently, multiple antenna based communications systems are reported to increase spectral efficiency and to improve the link reliability [1]. While such multiple input multiple output (MIMO) systems are in their infancy, the growing demand for high throughput in communication systems will make them an integral part of future wireless communication systems.

The MIMO technology utilizing the orthogonal frequency division multiplexing (OFDM) scheme will be the basis of the future IEEE 802.11n standard [2]. The combination of these two technologies will enable transfer rates over $100 \mathrm{Mbps}$ which represents a two-fold increase over the current $802.11 \mathrm{a} / \mathrm{g}$ WLAN standard. However, these improvements lead to various manufacturing challenges, such as increased $\mathrm{RF}$ test cost. The increase in the number of RF signal paths with the MIMO technology will increase the RF test overhead considerably. In addition, various specifications, such as the mismatches between the RF branches of the transceiver, will need to be measured to meet the stringent performance requirements of the overall system. These challenges necessitate the allocation of more test resources compared to single input single output (SISO) systems.

In this paper, we propose a novel MIMO test technology that enables MIMO RF testing on a lower cost mixed-signal tester by using special configurations on the tester board. We take a multi step approach where the basic channel parameters, such as gain and $I I P_{3}$ are measured using the proposed low cost test architecture first, and these parameters are utilized to deembed remaining parameters from the measurements. We first characterize the transmitter and then use its parameters to test the receiver. We measure the gain, third order input intercept point $\left(I I P_{3}\right)$, and phase mismatch between the RF paths by introducing a modular arrangement of down conversion mixers on the test board. Our technique requires only a single RF frequency synthesizer to drive the downconversion mixers and obviates the need for RF analyzers. We use a frequency multiplexing scheme to combine the information into a single time domain signal to avoid switching whenever possible. Experimental results show that our technique can be used to measure the most significant specifications of the MIMO systems accurately and efficiently. This work constitutes a first attempt to reduce the test cost for MIMO systems by using circuitry on the load board with satisfactory accuracy for go/no-go testing.

\section{RELATED WORK}

Since testing all circuit specifications is not feasible due to time constraints, test engineers typically select a subset of these specifications, such as gain, $I I P_{3}$, and sensitivity. The test sets are determined through empirical characterization data [3] or through more detailed simulation based analysis [4][5].

The general trend in the RF test domain is to transfer most of the RF test functionality onto the tester boards to utilize mixed-signal testers instead of RF-enabled testers. In [6], an RF signal source based on an all-digital PLL bank is presented. The source is capable of generating single-tone, multi-tone, and modulated RF waveforms making it useful for reducing the cost of $\mathrm{RF}$ receiver testing where the test input is at the high frequency domain and the output response is in the low frequency domain. In [7][8][9], the authors propose to push most of RF tester functionality onto the tester boards, thereby generating a specialized RF test equipment out of an ordinary mixed-signal tester. In [10], multi site testing of the GSM/GPRS RF transceivers is presented.

In order to reduce the test time, an arrangement of RF sources, mixers, combiners, splitters, and relays are utilized to shift most of the tester functionality into the load board. In order to simplify the load board designs, a popular alternative 

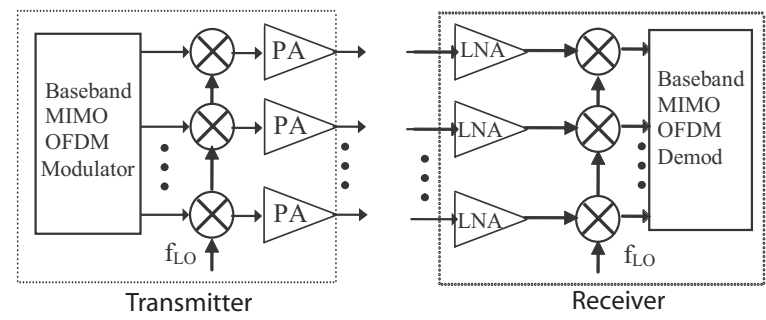

Fig. 1: Simplified NxN MIMO Transceiver Architecture

is to utilize on-chip implementations of these components. Peak and RMS detectors have been popular on-chip circuit components to obviate the need for RF testers. In [11], an on-chip implementation of embedded feature extractors are discussed. Several sensors are placed along the signal path which produce DC or low frequency output signals. In [12], the authors present a BiST technique composed of peak detectors, signal forwarding switches, and a test amplifier to characterize the specifications of a low noise amplifier (LNA).

\section{MIMO SYSTEM OVERVIEW}

The emerging IEEE $802.11 \mathrm{n}$ communication standard is based on the OFDM modulation scheme and a MIMO architecture [2]. OFDM modulation utilizes multiple sub-carriers in a wide channel. OFDM receivers are typically implemented with low intermediate frequency (IF) or zero-IF frequency configuration as DC offsets are easily removed without concern of losing data. In this work, we develop a generic test method for the measurement of the parameters that are most likely to affect the system performance based on the OFDM modulation scheme. However, this method can be applied to all MIMO systems regardless of the modulation scheme.

In the MIMO technology, the RF signal paths utilize the same frequency band, and carry independent data streams. Figure 1 depicts a simplified NxN MIMO architecture with $N$ transmit and receive antennas. Since the same frequency band is utilized, only one frequency synthesizer is needed.

A number of MIMO implementation approaches target various improvements such as increasing the power efficiency by maximizing the diversity gain, and layering to increase the channel capacity. However, all the implementations utilize digital signal processing blocks to recover the transmitted data effectively. This includes time synchronization, frequency offset estimation, and correction [13][14].

\section{A. RF impairments}

Impariments in the RF signal path such as compression and phase difference between the RF branches degrade the channel efficiency [2][15][16].

Linearity of the RF signal path is an important requirement for the OFDM based systems since the data is conveyed both on the amplitude and the phase of the system. While MIMO implementations inherent these functional requirements, the deviations among the parameters of the RF signal paths may also affect the overall system quality. The RF impairments that impact the MIMO operation the most can be summarized as gain and phase imbalances between the RF signal paths and non-linearity [16]. Since MIMO systems reconstruct the transmitted signal through digital signal processing and incorporate spatial diversity into the calculations, the differences may corrupt the transmitted data or degrade the link quality.

\section{B. MIMO Test Challenges}

With the introduction of MIMO systems, the test concern in terms of the instrumentation cost will be even more prononced due to the need to excite and analyze multiple RF signal paths. One alternative is to test each branch sequentially by switching a single RF source and a single analyzer from one branch to another. Using this sequential approach, the test time for one NxN MIMO system is at least $\mathrm{N}$ times that of a SISO system. This time may become longer with the addition of the RF switching times and instrument settling times which are typically in the range of $10-80 \mathrm{~ms}$ per switching [17]. Moreover, one of the most significant factors of the RF performance, the phase difference among the RF branches, cannot be accurately determined through a sequential test, since it requires synchronized analysis.

Another alternative is concurrent testing of all branches using multiple RF sources and analyzers. However, high cost of such test equipment would prohibit such an approach. In addition, the measurement of phase mismatch requires synchronized analysis of multiple RF branches, which requires specialized equipment increasing the test cost further.

\section{Test Methodology}

In this work, we aim to reduce the test resource usage of MIMO circuits through both reducing the test time and obviating the need for RF enabled instrumentation for go/nogo testing. We consider vital RF performance parameters such as path gain, $I I P_{3}$, and the phase mismatch among the RF branches. Although our approach can be applied to extract more complex parameters, such as spectrum mask, these parameters are typicaly not measured in the final production testing due to test time concerns.

A cost efficient alternative to the signal analysis at RF frequencies is to utilize low frequency signals for testing. In order to perform the necessary frequency conversion operation, we use on-board downconversion mixers. The on-board mixers, similar to any other on-board components, will be fully characterized to prevent their process variations or defects from jeopardizing the test quality. Full characterization of the on-board mixers can be attained at a relatively low cost during the load board design phase.

Our test methodology consists of several steps. At each step, we use the results from the prior measurements to deembed the parameters of interest. Whenever possible, we avoid sequential testing of the paths by combining the output signals. When sequential testing is necessary, we use low frequency analog signal multiplexing to avoid long mechanical 


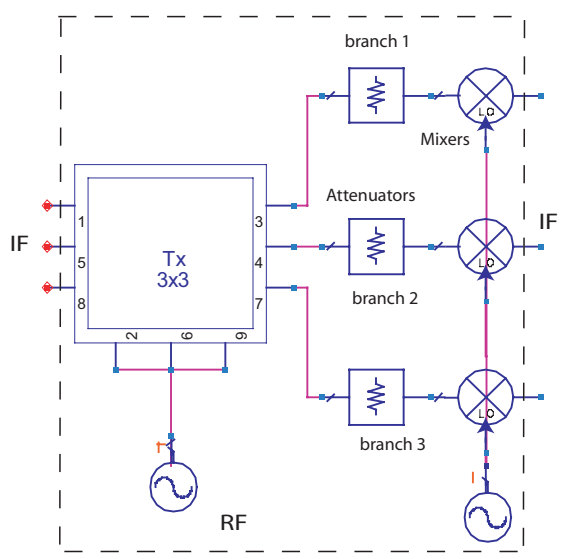

Fig. 2: Transmitter gain and $I I P_{3}$ measurement setup

RF switching times. The steps of our test methodology are as follows:

- Gain and $I I P_{3}$ measurement for the transmitter (TX) using frequency domain multiplexing for the RF branches.

- Phase mismatch measurements between pairs of TX paths using the gain information and time domain analog signal multiplexing at the output.

- Gain and $I I P_{3}$ measurements for the receiver (RX) using the TX as the RF signal generator and frequency domain multiplexing of low frequency analog signals.

- Phase mismatch measurements between the pairs of the RX paths using the gain information and analog signal multiplexing at the output of the RX.

\section{A. TX Gain and $I I P_{3}$ Measurements}

Traditional TX testing utilizes low frequency analog signals as the input stimuli and analyze the RF response of the circuit. Since RF signal analysis typically requires much more expensive equipment, we propose to downconvert the TX output to the low frequency domain by fully characterized, down conversion mixers on the load board, as depicted in Figure 2. In order to enable the measurements of the inherent non-linearity and to keep the stimuli generation simple, we utilize two tone signals as input stimulus. In order to reduce the test cost further by reducing the test time, we propose to use a frequency domain multiplexing scheme for distinct TX paths. We then combine the outputs of the downconversion mixers to generate a single analog signal for analysis. The frequency synthesizer is still set to a particular channel, and is driving all the upconversion mixers inside the circuit under test (CUT). As OFDM channels are allocated with wider bandwidth (20 $\mathrm{MHz}$ ) compared to other standards (e.g. $1 \mathrm{MHz}$ for Bluetooth [18], $200 \mathrm{kHz}$ for GSM [10]), we can safely place multiple two tone signals with a channel to enable concurrent gain and $I I P_{3}$ measurements for all the RF branches.

Since a single frequency synthesizer drives all the RF branches inside the CUT, the frequency domain multiplexing needs to be done at the input of the TX. Fortunately, generating multiple low-frequency analog signals is much easier and can

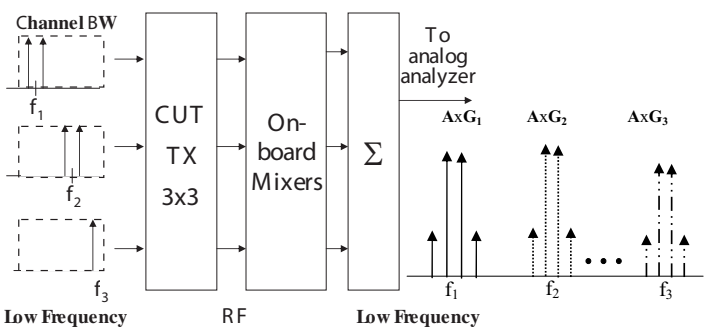

Fig. 3: The combination of the outputs of the on-board mixers and the resulting combined output in the frequency spectrum

be done through direct digital synthesis if the tester does not support multiple distinct analog outputs. For integrated RF components, the necessary low frequency analog signals can be generated by the on-chip DSP and ADC.

In order to characterize and distinguish each RF branch individually, and to take the advantage of parallel testing, we adjust the frequencies of the input stimuli such that the higher order intermodulation terms do not interfere with the fundamental tones. Figure 2 illustrates the input enabled frequency domain multiplexing scheme for concurrent testing of all TX RF branches. By measuring the signal power at the individual frequency locations for each branch, the path gain and $\mathrm{IIP}_{3}$ can be concurrently measured. Since the downconversion mixers on the load board are fully characterized, the gain and the $I I P_{3}$ parameters can be de-embedded from the measured values. The gain of the TX paths can be linearly de-embedded from the overall measured gain. To de-embed the $I I P_{3}$ of the path, we utilize the measured gain of the TX paths, the characterized gain and $I I P_{3}$ of the on-board mixer and the cascaded $I I P_{3}$ formulation [19] as given in Equation 1:

$$
\frac{1}{A I P_{3, \text { measured }}^{2}}=\frac{1}{A I P_{3, D U T}^{2}}+\frac{G_{D U T}}{A I P_{3, \text { mixer }}^{2}}
$$

where $A I P_{3, \text { measured }}$ is the measured $I I P_{3}$ at the output of the downconversion mixer, $G_{D U T}$ is the gain of the Device Under Test (DUT), and $A I P_{3, \text { mixer }}$ is the characterized $I I P_{3}$ of the downconversion mixer.

\section{B. TX Phase Mismatch Measurements}

In addition to the gain and $I I P_{3}$ measurements of the individual branches, the amount of the phase difference is an important factor that determines the overall quality of the MIMO system. The important point to note is that, the absolute phase shift of an RF branch is not a damaging factor for the MIMO performance. However, the difference between the phases of the RF branches impacts the TX performance negatively. Traditionally, measuring the phase difference between the RF signal paths necessitates the use of an RF analyzer capable of synchronized sampling of two distinct RF signals. In this work, we utilize a novel way to measure the amount of the phase difference between the RF branches.

We observe that the phase difference between two identical frequency RF signals reflects as a DC signal when the two signals are multiplied though a mixer. However, this DC 


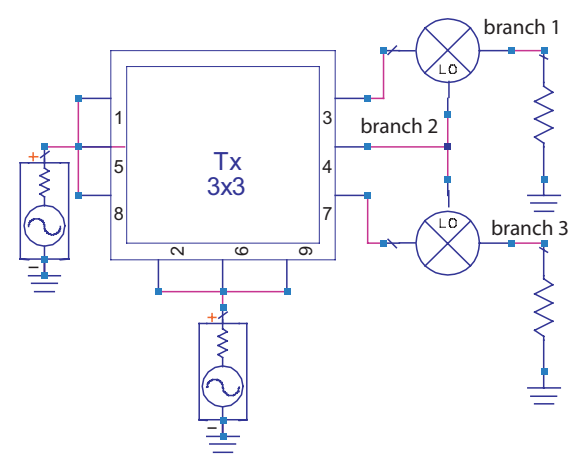

Fig. 4: Test setup for phase mismatch measurement

signal will be corrupted by other DC offsets of the system as well as DC blocking mechanisms of the system and the test setup. Therefore, we need to shift the term containing the phase difference to higher frequencies where no fundamental is present. Fortunately, we can provide this frequency shift by utilizing two tone signals instead of single tone signals. As a result, our phase mismatch measurement technique is based on using a single two-tone low frequency analog input and mixing the RF outputs, as shown in Figure 4. Both branches, whose phase mismatch is to be measured, receive identical input signals. Therefore, the branches process the same signal at the same time, enabling accurate phase difference characterization.

When a two tone input is applied, both of the output tones of the particular branch will have the same absolute phase shift. When the output of a branch is multiplied by the output response of the other branch, the cross tones will be multiplied. Figure 5 depicts the mixing operation in the frequency domain.

The mathematical formulation for this mixing operation can be obtained as follows:

$$
\begin{aligned}
V_{\text {branch }_{i}}= & A G_{i}\left\{\cos \left(\omega_{1} t+\phi_{i}\right)+\cos \left(\omega_{2} t+\phi_{i}\right)\right\} \\
V_{\text {mix }}= & G_{\text {mix }} \times V_{\text {branch }_{1}} \times V_{\text {branch }} \\
= & A G_{1}\left\{\cos \left(\omega_{1} t+\phi_{A}\right)+\cos \left(\omega_{2} t+\phi_{A}\right)\right\} \times \\
& G_{\operatorname{mix}} A G_{2}\left\{\cos \left(\omega_{1} t+\phi_{B}\right)+\cos \left(\omega_{2} t+\phi_{B}\right)\right\} \\
= & G_{\operatorname{mix}} A^{2} G_{1} G_{2}\left\{\cos \left(\omega_{1} t+\phi_{A}\right) \cos \left(\omega_{1} t+\phi_{B}\right)\right. \\
& +\cos \left(\omega_{1} t+\phi_{A}\right) \cos \left(\omega_{2} t+\phi_{B}\right) \\
& +\cos \left(\omega_{2} t+\phi_{A}\right) \cos \left(\omega_{1} t+\phi_{B}\right) \\
& \left.+\cos \left(\omega_{2} t+\phi_{A}\right) \cos \left(\omega_{2} t+\phi_{B}\right)\right\}
\end{aligned}
$$

$$
\begin{aligned}
V_{\text {mix }}=\frac{G_{\text {mix }} A^{2} G_{1} G_{2}}{2}\left\{2 \cos \left(\left|\omega_{2}-\omega_{1}\right| t+\left|\phi_{B}-\phi_{A}\right|\right)\right. \\
+\cos \left(2 \omega_{1} t+\left(\phi_{A}+\phi_{B}\right)\right) \\
+\cos \left(2 \omega_{2} t+\left(\phi_{A}+\phi_{B}\right)\right) \\
\left.+2 \cos \left(\left(\omega_{1} t+\omega_{2} t\right)+\left(\phi_{A}+\phi_{B}\right)\right)+D C\right\} \quad \text { (4) }
\end{aligned}
$$

.

the paths reflects as a coefficient for the cross products of the input signal tones. Since the input amplitude as well as the gains of each path are known, the phase difference can be calculated using the power and the phase of the tone at the difference frequency, $\left|\omega_{1}-\omega_{2}\right|$.

Some testers may require additional steps to obtain the amount of phase difference from the instrument. The two tone signal generator and the sampler should be driven from the same reference clock, and the amount of time offset should be calibrated before making the measurements.

\section{RX Tests}

The RX performs the complimentary operation of the TX, recovering the transmitted signal and down converting it to the IF frequencies. Since the IC that contains a defective TX is pruned away from the conveyor belt, a low cost test methodology primarily targeting go/no go testing can be achieved by utilizing the TX through a loopback connection to test the RX. Typically OFDM receivers are implemented with a low (or zero) intermediate frequency. Therefore, the RX-TX frequency offset problem that is common to loopback testing is not an issue for OFDM systems. Since the TX is characterized before the RX is tested, the performance parameters of the TX can be de-embedded from the overall results to characterize the RX. Figure 6 depicts the test setup for RX testing. The output of the TX is connected to the RX through attenuators in order to prevent compression.

The same two step approach that is utilized to measure the gain, $I I P_{3}$, and the phase difference among the signal paths of the TX can be applied to estimate corresponding parameters for the RX. Even though possible accumulated inaccuracies from the TX testing propagate into the RX parameter extraction, the information obtained through this method is more than satisfactory for go/no go testing. In the following section, we evaluate the overall impact of the accumulating errors on a transistor level MIMO transceiver circuit.

\section{EXPERIMENTAL RESULTS}

In the experiment, our goal is to evaluate the accuracy of our measurement set-up. The accuracy loss may originate from

where $A$ is the amplitude of the input signal, $\omega_{1,2}$ are the tone
frequencies that constitute the two tone signal, $G_{m i x}$ is the
linear gain coefficient of the on-board mixer, and $\phi_{A, B}$ are
the phases of the RF branches. The phase difference between

where $A$ is the amplitude of the input signal, $\omega_{1,2}$ are the tone
frequencies that constitute the two tone signal, $G_{m i x}$ is the
linear gain coefficient of the on-board mixer, and $\phi_{A, B}$ are
the phases of the RF branches. The phase difference between

where $A$ is the amplitude of the input signal, $\omega_{1,2}$ are the tone
frequencies that constitute the two tone signal, $G_{m i x}$ is the
linear gain coefficient of the on-board mixer, and $\phi_{A, B}$ are
the phases of the RF branches. The phase difference between

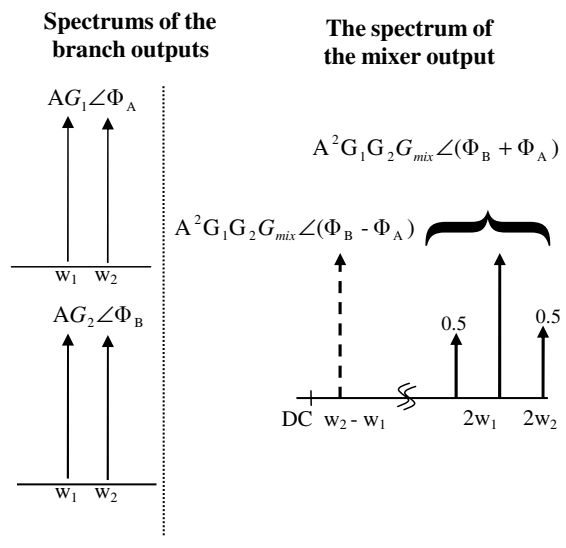

Fig. 5: Mixing of the tones of the adjacent branches 


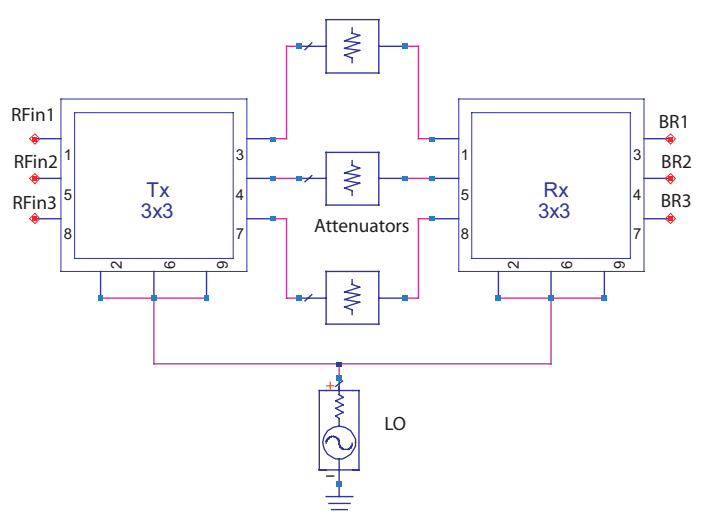

Fig. 6: Receiver Test using the transmitter as a test vehicle

two sources. The first source is due to the finite accuracy of the spectrum analysis, which generates random errors in each measurement step. The second source of error is the accumulation of uncorrelated errors during each measurement setup until the final step. In this section, we present the test accuracy results based on non-ideal on-board mixers, detailed transistor level simulations that include the effects of process variations, and the loss in accuracy at each step due to the inaccuracy of the preceding setup.

\section{A. MIMO RF Transceiver Design}

The MIMO transceiver consists of three identical RX and TX chains operating at $5.3 \mathrm{GHz}$ frequency band. The TX is modeled using a transistor level double balanced mixer and a power amplifier with adjustable gain, noise figure, $I I P_{2}$, and $I_{I} P_{3}$ parameters. The frequency synthesizer that drives the mixers is modeled as a single monolithic oscillator which is enhanced by the addition of in-band phase noise $(-60 \mathrm{dBc} / \mathrm{Hz}$ nominal) and spurs $(-58 \mathrm{dBc} / \mathrm{Hz}$ nominal). The $\mathrm{RX}$ consists of transistor level LNA and mixer circuits. The LNA is a stability optimized version of the cascode LNA architecture [20][4]. The process node is $80 \mathrm{~nm}$ and BSIM4 models are utilized for simulation. All circuits are designed and simulated at the transistor level using ADS (Advanced Design System). The LNA and the mixer circuits are given in Figure 7.

The onboard downconversion mixers are modelled by using built in mixers of the ADS. The mixers have a conversion gain $(-7.3 \mathrm{~dB}), I I P_{3}(10 \mathrm{dBm})$ and $1-\mathrm{dB}$ compression point $(-1$ $\mathrm{dBm})$ parameters that are set to represent off the shelf mixers.

In order to verify our proposed test approach, we created 200 instances of the nominal circuit by allowing $10 \%$ variation in the resistor $(R)$, capacitor $(C)$, inductor values $(L)$, and transistor length $\left(L_{e f f}\right)$ and widths $(W)$ through Monte-Carlo simulations. There are 51 parametric variations for each TX

TABLE I: Summary of the transceiver specifications

\begin{tabular}{|c|c|c|c|}
\hline & Gain $(\mathrm{dB})$ & $I I P_{3}$ & Phase \\
\hline Transmitter & $21 \mathrm{~dB}$ & $-4 \mathrm{dBm}$ & $-65^{\circ}$ \\
\hline Receiver & $11 \mathrm{~dB}$ & $-18 \mathrm{dBm}$ & $107^{\circ}$ \\
\hline
\end{tabular}

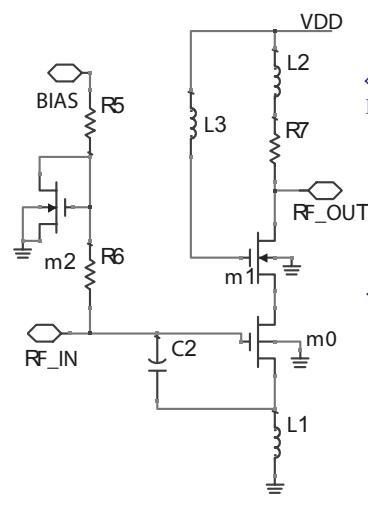

(a) LNA

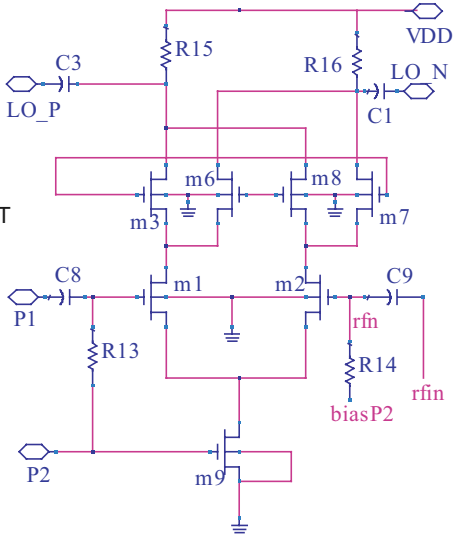

(b) Mixer
Fig. 7: Transistor level LNA and Mixer circuits

branch and 105 parametric variations for each RX branch. Table I depicts the TX and RX branch specifications. The branches are designed to be identical; ideally the phase difference between the branches are zero.

\section{B. Test Accuracy Results}

From all the randomly generated circuit instances, we conduct two sets of measurements. First we analyze the direct output of each branch and measure the desired parameters (gain, $I I P_{3}$, phase mismatch). Then we repeat the measurement with our test methodology. We compare the results of the measurement for each randomly generated circuit instance and extract an error histogram for all the parameters.

Figure 8 depicts the error histograms of the gain, $I I P_{3}$, and phase difference measurements for transmitter (Panels A through $\mathrm{C}$, respectively), and the receiver circuits (Panels D through F, respectively). As the transmitter is utilized a test vehicle for the receive chain, the propagation of the error can be observed by Panel A to Panel D, where the centroid of the histogram moves to a higher value. A similar trend is observed for the $I I P_{3}$ and phase difference measurements. Even though the inaccuracy of the receiver circuit is higher compared to the transmitter accuracy, it is still at very low levels.

\section{CONCLUSION}

In this paper, we present a MIMO set strategy that obviates the need for RF enabled analyzers while enabling the measurement of critical parameters of the RF path. We utilize a multi step approach to test the complete system starting with the transmitter and later use the transmitter as a test device for the receiver. At each step of the test process, current parameters of interest are de-embedded from the measurements using the results of preceding measurement.

We develop a novel test setup to measure the phase mismatches between the RF branches accurately using various configurations of mixers on the load board. Experimental results indicate that even though a slight inaccuracy propagates through the de-embedding process, the critical parameters of the overall system can be measured with a high accuracy. 


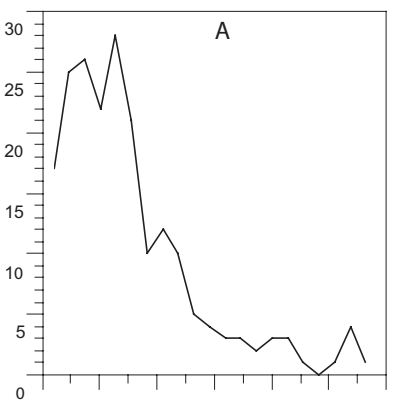

$\begin{array}{llllllll}0.000 & 0.002 & 0.004 & 0.006 & 0.008 & 0.010 & 0.012 & 0.1\end{array}$
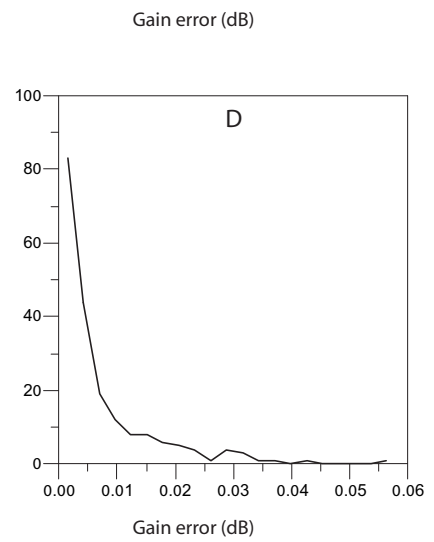

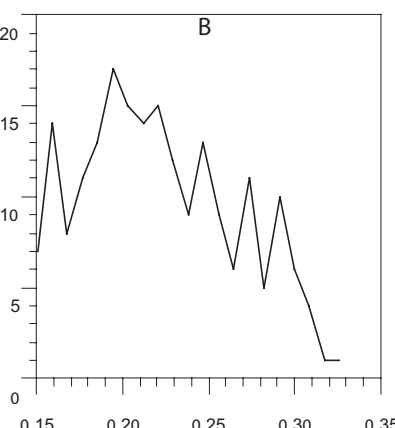

IIP3 error (dB)

Receiver Error Histograms

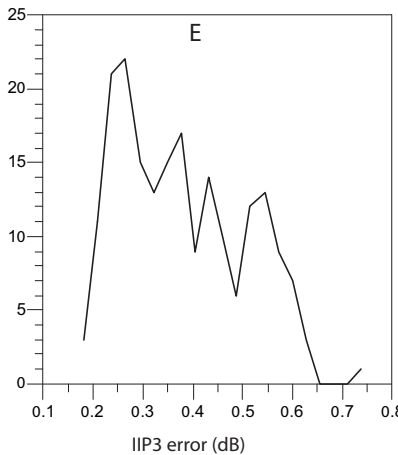

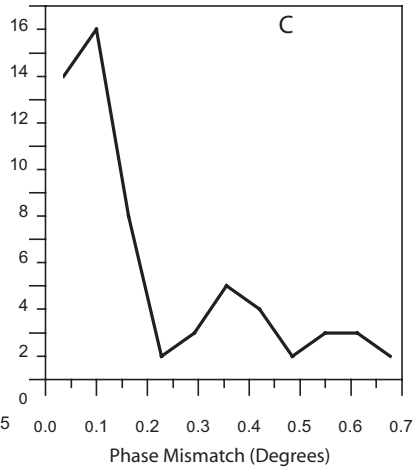

Phase Mismatch (Degrees)

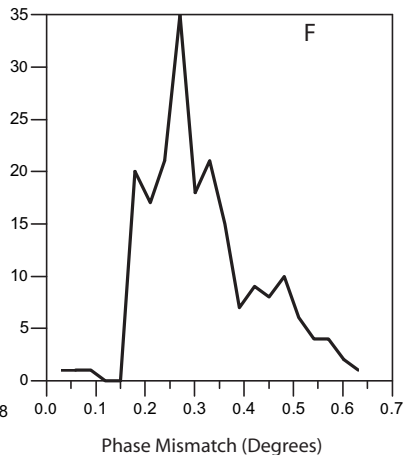

Fig. 8: Error histograms for gain, $I I P_{3}$, and phase mismatch measurements

This work constitutes a first attempt to reduce the test cost for MIMO systems by using circuitry on the load board with a satisfactory accuracy for go/no-go testing.

\section{ACKNOWLEDGMENT}

This work is supported by the Semiconductor Research Corporation under the contract number 2004-TJ-1247 and by National Science Foundation under contract numbers CCF0545456 and CCF-0540994.

\section{REFERENCES}

[1] H. Bolcskei and M. Ibnkahla, CRC Handbook on Signal Processing for Communications, 2004.

[2] S. Woo, D. Lee, K. Kim, Y. Hur, C.-H. Lee, and J. Laskar, " Combined effects of RF impairments in the future IEEE 802. 11n WLAN systems," in Vehicular Technology Conference, June 2005, pp. 1346 - 1349.

[3] S. Huss and R. Gyurcsik, " Optimal ordering of analog integrated circuit tests to minimize test time," in Design Automation Conference, June 1991, pp. $494-499$.

[4] E. Acar and S. Ozev, "Parametric test development for RF circuits targeting physical fault locations and using specification-based fault definitions," in International Conference of Computer Aided Design, Nov 2005, pp. $73-79$.

[5] F. Liu and S. Ozev, "Fast Hierarchical Process Variability Analysis and Parametric Test Development for Analog/RF Circuits," in International Conference on Computer Design, Oct 2005, pp. 161 - 170.

[6] I. T. Sylla, "Building an RF source for low cost testers using an ADPLL coltrolled by Texas Instruments digital signal processor DSP TMS320C5402," in IEEE International Test Conference, Nov 2003, pp. $659-664$.

[7] J. Ferrario, R. Wolf, S. Moss, and M. Slamani, "A low-cost test solution for wireless phone RFICs," IEEE Communications Magazine, pp. 82-88, September 2003.
[8] J. Ferrario, R. Wolf, and H. Ding, "Moving from mixed-signal to RF test hardware development," in IEEE International Test Conference, Nov 2003, pp. 948-956.

[9] D. Brown, J. Ferrario, R. Wolf, J. Li, and J. Bhagat, "RF Testing on a Mixed Signal Tester," in IEEE International Test Conference, 2004, pp. $793-800$.

[10] L. Zhang, D. Heaton, and H. Largey, "Low cost multisite testing of quadruple band GSM transceivers," in IEEE International Conference on Test, Nov 2005, pp. $405-411$.

[11] S. Bhattacharya and A. Chatterjee, " Use of embedded sensors for builtin-test RF circuits," in IEEE International Conference on Test, Nov 2005, pp. $801-809$.

[12] J. Ryu, D. Kadam, T. Alex, and B. Kim, "Design of a new RF BIST Circuit for $5.25 \mathrm{GHz}$ Low Noise Amplifiers," in Bipolar/BiCMOS Circuits and Technology, 2004, pp. 257-260.

[13] G. Stuber, J. Barry, S. McLaughlin, Y. Li, M. Ingram, and T. Pratt, “ Broadband MIMO-OFDM wireless communications," in Proceedings of the IEEE, vol. 92, Feb 2004, pp. $271-294$.

[14] A. Mody and G. Stuber, "Synchronization for MIMO OFDM systems," in IEEE Global Telecommunications Conference, Nov 2001, pp. 509513.

[15] J. Liu, A. Bourdoux, J. Craninckx, P. Wambacq, B. Come, S. Donnay, and A. Barel, " OFDM-MIMO WLAN AP front-end gain and phase mismatch calibration," in IEEE Radio and Wireless Conference, Sept 2004, pp. $151-154$.

[16] N. Khaled, S. Jagannathan, A. Bahai, F. Petre, G. Leus, and H. De man, "On the impact of multi-antenna RF transceivers' amplitude and phase mismatches on transmit MRC," in IEEE International Conference on Acoustics, Speech, and Signal Processing, March 2005, pp. 893-896.

[17] "Agilent 83236B PCS Interface Documentation," http://www.agilent.com, 2006.

[18] L. Nord, "Specifications of the Bluetooth system," http://www.bluetooth.org, 2001.

[19] B. Razavi, RF Microelectronics. Prentice-Hall, 1998.

[20] T. Lee, The Design of CMOS Radio-Frequency Integrated Circuits. Cambridge University Press, 2004. 